\title{
Overview of long non-coding RNA and its role in breast cancer
}

\author{
Elda A. Flores-Contreras and Carlos Córdova-Fletes* \\ Department of Biochemistry and Molecular Medicine, School of Medicine, Autonomous University of Nuevo Leon, Monterrey, Nuevo Leon, Mexico
}

\begin{abstract}
The mammary glands are made of different cell populations that have great cellular plasticity, which is mainly regulated by epigenetic changes that allow the expression of specific transcripts needed depending on the stage of development (embryonic, puberty, pregnancy, lactation, involution, and postmenopause). Therefore, it is important to maintain strict regulation at each stage because aberrations in epigenetic regulation can lead to breast cancer. Among the epigenetic regulators are long non-coding RNAs (IncRNAs), which play important roles during the development of the breasts. Further, they can be used as biomarkers in breast cancer since their expression and emergence correlate with the patient's survival, as well as the aggressiveness of the tumor; the IncRNAs lead to epigenetic changes and the activation of signaling pathways, causing an accelerated rate of proliferation, metastasis, and evasion of the action of chemotherapeutic agents. Therefore, IncRNAs may be potential therapeutic targets. In this review, we show examples of IncRNAs that exert resistance on the different subtypes of breast cancer, through the activation of signaling pathways protein kinase $b(A K T)$, the extracellular signal-regulated kinases (ERK), the mammalian target of rapamycin (mTOR), and (wingless/integrated [Wnt])/(beta-catenin). Some of these pathways are shared among the different subtypes, allowing cancer cells to activate the same pathways through different mechanisms, promoting tumor growth and resistance to various therapeutic agents. Furthermore, the mechanisms by which IncRNAs help acquire resistance and strategies to determine their function are presented in this review.
\end{abstract}

Key words: Long non-coding RNAs. Epigenetics. Genetic mutations. Resistance.

\section{Introduction}

Micro- and macro-anatomy of mammary glands suffer drastic morphological, anatomic, and epigenetic modifications during embryonic development, puberty, pregnancy, lactation, involution (ceasing of milk production), and postmenopause (Fig. 1). These modifications are caused by epigenetic indicators, which may be extreme conditions (environment and nutrition) or signals specific to development (differentiation) ${ }^{1}$ which cause changes at a biochemical level through post-transcriptional modifications observed in chromatin's non-histone proteins ${ }^{2-4}$, long non-coding RNAs (IncRNAs) ${ }^{5}$, and histones (methylated - turning off or acetylated - turning on target genes) $)^{2,6}$ (Fig. 1). These alterations lead to the remodeling of chromatin, allowing the activation or inhibition of transcription in certain genes, leading to morphological and functional changes. These modifications occur in the different cellular populations of the mammary glands.

Mammary glands are made up of adipocytes, epithelial, endothelial, and stromal cells, which converge and communicate simultaneously through hormones, growth factors, and cytokines, ensuring the correct organization of the tissue ${ }^{7}$ (Fig. 2).

The types of communication (activation of the different signaling cascades) which will be established

\section{Correspondence:}

*Carlos Córdova-Fletes

E-mail: carlos.cordovafl@uanl.edu.mx
Available online: 09-08-2019

Date of acceptance: 30-04-2019

DOI: 10.24875/RMU. 19000040
Medicina Universitaria. 2019;21(2):63-73 www.medicinauniversitaria.org CC BY-NC-ND license (http://creativecommons.org/licenses/by-nc-nd/4.0/) 


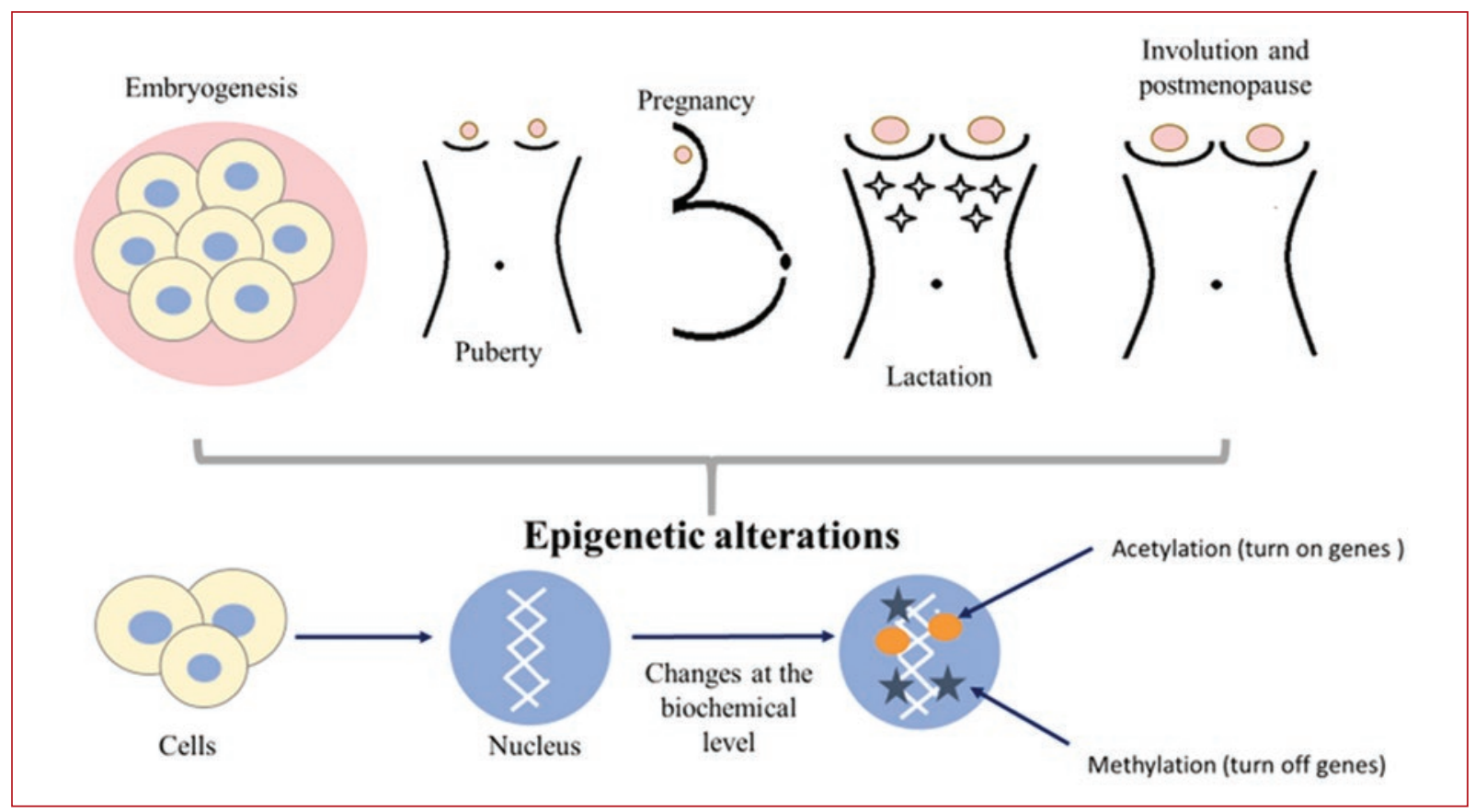

Figure 1. Epigenetic changes caused by the stages of development in the breasts. To fulfill its function, the mammary glands present changes in their expression patterns, caused by biochemical modifications in their DNA, characteristic of each stage of development.

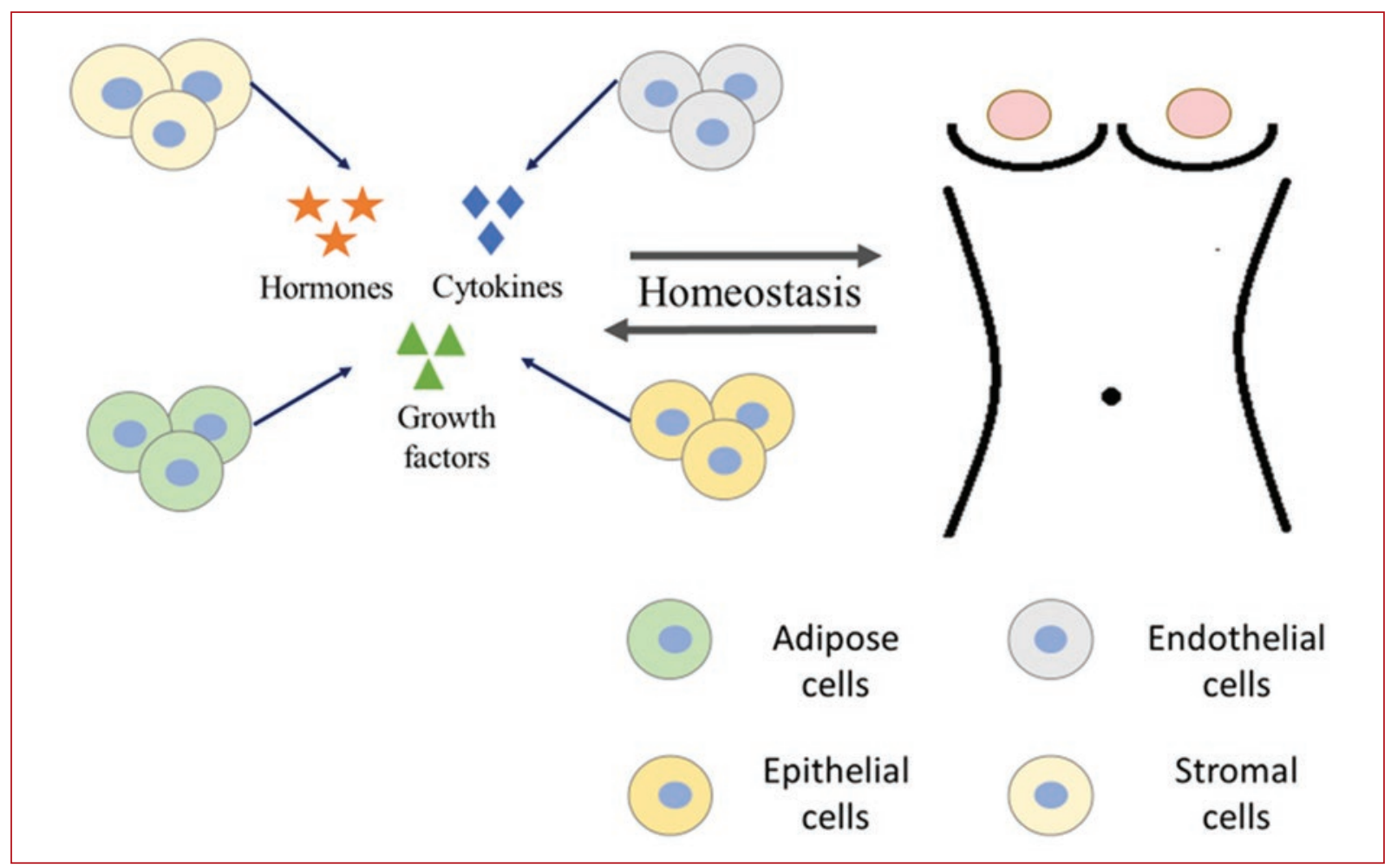

Figure 2. Communication between the different cellular populations of the breasts. The different populations of cells (adipocytes, endothelial, epithelial, and stromal) that make up the adult mammary glands constantly and simultaneously are in communication, through hormones, cytokines, and growth factors, to maintain the homeostasis of breast tissue. 
amongst the heterogeneous cellular population of the breasts will depend on the development stage they are at (embryonic, puberty, pregnancy, lactation, involution and postmenopause). For example, during embryogenesis, morphogenesis depends on the signals originating from mesenchyme (supporting tissues which surround organs) and from the absence of hormones ${ }^{8}$. On the other hand, during the puberty-adulthood transition, the main signals of communication are those hormones which emanate from the pituitary gland and the ovaries. These stimulate the formation of the parenchyma (essential tissue of the organs) and allowing the ramification of the milk ducts, the development of the acinus, and the increase of the adipocytes (main component of non-lactating mammary glands). The latter, express aromatase enzymes which help in the conversion of androgens into estrogens, establish a paracrine communication with epithelial cells through the estrogen receptors alpha (ER). It is worth noting that epithelial cells release vascular endothelial growth factor, which allows the regulation of angiogenesis. Moreover, the proliferation, differentiation (during morphogenesis), and cellular death of epithelial cells are regulated by fibroblasts (another main component of mammary glands). These are in charge of maintaining, remodeling, and degrading the extracellular matrix (metalloproteinase) $)^{7}$. During pregnancy, progesterone and prolactin are secreted, promoting the formation of mammary alveolus, which will secret milk during lactation ${ }^{8}$; while the involution of mammary glands leads to changes in the architecture of cellular populations, causing degradation and remodeling of the extracellular matrix. In addition, alveolar cells undergo an apoptosis. Even though the way this occurs is not fully dilucidated, it is suggested to be a result of an inflammatory response which most likely is caused by the formation of $\alpha$-lactalbumin (a component of milk) ${ }^{9}$. Finally, during the postmenopausal stage, the second mammary involution occurs, which is linked to a decrease in the functional activity of the ovaries, causing a remodeling of mammary glands including a decrease in mammary tissue and an increase in adipose tissue, which leads to a loss of function of this tissue ${ }^{9}$.

Mammary glands represent one of the more complex organs at a cellular level, due to the interactions (paracrine communication) between different cell populations. Moreover, each population brings its own cellular plasticity. Hence, the cells ought to maintain a fine epigenetic regulation, one which lets them activate the proper signaling pathways for each stage of development. Many of these processes are
Table 1. Similarities/differences between IncRNAs and transcripts that encode proteins

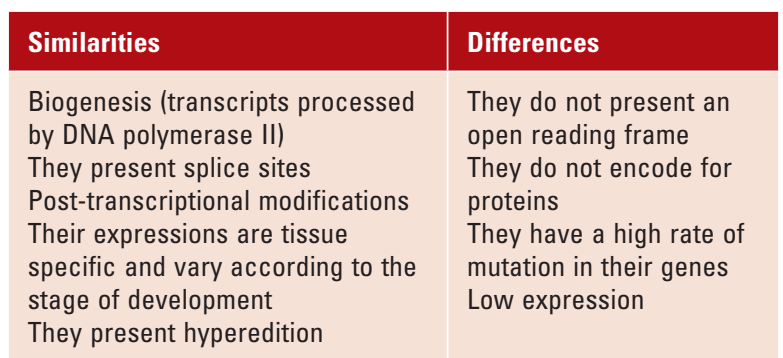

regulated by non-coding transcripts, among which are IncRNAs.

\section{Characteristics of IncRNA}

The number of genes which encode this kind of RNA remains uncertain, yet estimations suggest a range between 20,000 and 50,000 genes ${ }^{10,11}$. These genes transcribe products $>200$ nucleotides of length. Those transcripts present characteristics very similar to messenger RNAs (mRNAs) regarding their biogenesis since the transcription process is carried out by the RNA polymerase II under a combination of different factors and transcription regulators, which join promoters and potentiators, present alternative splicing and post-transcriptional modifications (polyadenylation and hyperedition) $)^{12-14}$. In addition, its expression patterns just the genes which encode proteins and specific tissue and vary depending on the stage of development ${ }^{15}$. On the other hand, the differences between IncRNAs and mRNAs are the lack of an open reading framework, and its expression tends to be lower ${ }^{12,16}$.

In addition, IncRNA genes have a high evolution rate concerning those genes which encode proteins since they evolve neutrally and the selection force exercised is low, indicating that many of these transcripts are not functional (Table 1). The amount of IncRNA sequences preserved by natural selection is lower than $5 \%{ }^{17}$.

However, at the sequence level, IncRNAs do not present preserved regions. On the contrary, their secondary structure is well preserved, allowing them to maintain their function ${ }^{18}$. A clear example is provided by the IncRNA BORG (BMP/OP-Responsive Gene), which promotes the development of breast cancer in murine at an accelerated proliferation and metastasis rate, and when transfected in MCF-7 cells (breast cancer cellular line with a low BORG expression), changes 


\section{A Intergenic IncRNAs}

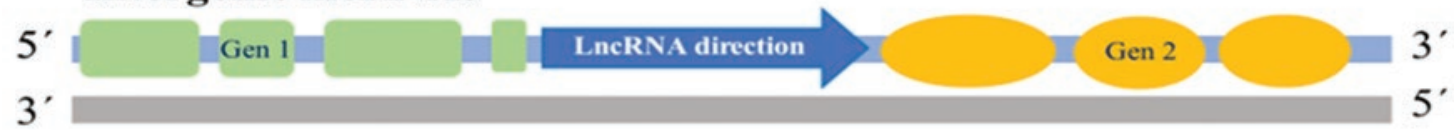

B Intronic IncRNAs
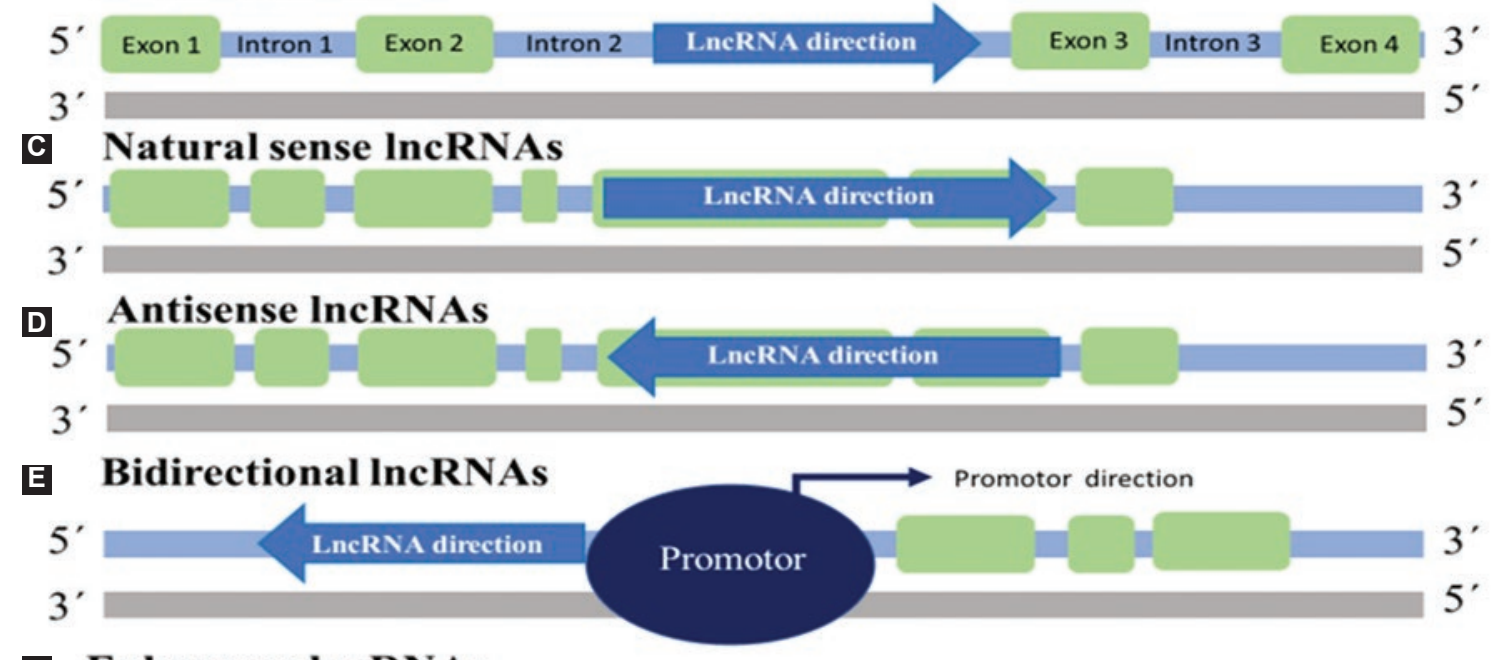

F Enhancers IncRNAs

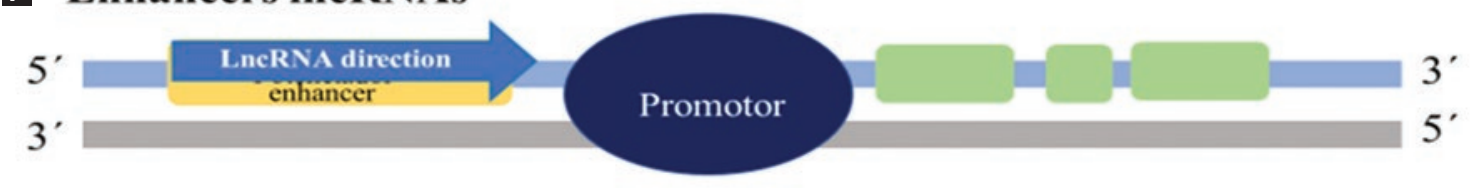

Figure 3. Genomic organization of long non-coding RNAs (IncRNAs). IncRNAs are classified according to their location in the genome; A: which can be present between two genes, which are called intergenic IncRNAs; B: or they may be located in introns (between two exons) of genes encoding proteins which are called intronic IncRNAs; C: They can also be located in the coding regions, that is, in the exons, and overlap in the same direction of the genes that encode proteins or in the opposite direction, these being called natural sense;

D: or antisense; IncRNAs, respectively; E: On the other hand, bidirectional IncRNAs use the promoters of the genes that encode proteins but go in the opposite direction; F: and finally, the enhancing IncRNAs are located in enhancing elements (regulatory elements of the expression of genes that encode proteins).

in growth and inhibition of programmed death (apoptosis) can be seen ${ }^{19}$.

Based on their function, IncRNAs are divided into two kinds, constitutive or regulatory. IncRNAs with constitutive functions are constantly expressed and are involved in vital processes for the cell. IncRNAs with regulatory functions are expressed during development ${ }^{7}$. IncRNAs can be located in different parts of the genome, i.e. (a) intergenic IncRNAs exist between two genes which encode a protein; (b) intronic IncRNAs are produced from the intron of a gene; (c) natural sense IncRNAs are overlapping in the same direction with exons of genes that encode proteins; (d) antisense IncRNAs overlap in the opposite direction to the exons which encode proteins; (e) bidirectional IncRNAs share the same sequence promoters which encode proteins but go in the opposite direction; and (f) enhancer IncRNAs come from enhancing regions located in genes which express proteins ${ }^{20}$ (Fig. 3).

In the mammary glands, IncRNAs are in charge of transcriptional regulation, processing and maturing of mRNAs, and epigenetic regulation through the recruitment of chromatin remodelers (modifying methylation and acetylation patterns of histones). For example:

- Nuclear paraspeckle assembly transcript 1 (NEAT1) is an intergenic IncRNA, which is involved in the regulation and processing of transcripts, since it is an essential component for the formation of paraspeckles (compartments located in the nucleus), which are in charge of kidnapping proteins with transcriptional activity (transcription factors) and hyperediting mRNAs (change of an - A adenine for 
an - I inosine). This IncRNA is important for the morphogenesis and lactating process in mice ${ }^{21}$.

- ZNFX1 antisense RNA 1 is transcribed in the opposite direction to the gene, which encodes the ZNFX1 protein (zinc finger NFX1-type containing 1). Thus, it is an antisense IncRNA. This IncRNA functions as a tumor suppressor; it presents a high expression in mammary tissue and regulates alveolar development and differentiation of epithelial cells $^{22,23}$. Moreover, it modulates the assembly, production, and maturation of the ribosomes ${ }^{24}$.

- Pregnancy-induced non-coding RNA is an intergenic IncRNA, which depends on the stimuli of the development for its expression (special and temporal) and interacts with the polycomb 2 repressor complex, inducing modifications in chromatin, mainly for the inhibition of transcript expressions which induce the differentiation of mammary alveolar cells and prevents milk production until delivery ${ }^{25}$.

Therefore, alterations in the expression of IncRNAs, which maintain cellular homeostasis or that is in charge of the regulation of processes such as embryogenesis, puberty-adult transition, lactation, mammary involution, or postmenopause, lead to the initiation and progression of tumors which may result in breast cancer. These IncRNAs may be exclusive of one or be implicated in different subtypes of breast cancer ${ }^{26}$.

\section{Subtypes of breast cancer}

Breast cancer is a very heterogeneous disease, which has allowed us to set a classification criteria based on transcriptional profiles (PAM 50) of genes that encode proteins, resulting in three subtypes of breast cancer; these three subtypes were found by Su et al. (2014). The same subtypes were found when making a classification based on IncRNAs. These findings can provide more personalized therapies.

Today, breast cancer is classified in the following main subtypes:

- Luminal (Luminal A and Luminal B)

Luminal $A$ expresses estrogen and progesterone receptors and presents a low expression of Ki67. Luminal B expresses estrogen and progesterone receptors, negative or positive for human epidermal growth factor receptor 2 (HER2) and presents a high expression of Ki67.

This subtype responds well to hormone treatments, which may be aromatase inhibitors (enzymes in charge of the biosynthesis of estrogens), selective modulators of ER (tamoxifen), and selective degraders of ER.

- HER2

HER2 presents an amplification and overexpression of the ERB oncogene (receptor) encode by the HER2 gene. Following its activation, it promotes proliferation, differentiation, angiogenesis, invasion, and metastasis. Today, trastuzumab, which is a monoclonal antibody against ERBB2, is the approved therapy to treat this subtype ${ }^{27}$.

- Triple negative or basal

Basal-type tumors, also called triple-negative breast cancer, do not present therapeutic targets, and only $20 \%$ of these tumors respond well to standard chemotherapy (therapies against RE) ${ }^{28}$.

\section{The role of IncRNAs in the different subtypes of breast cancer}

It is of great importance to know the mechanisms and functions of breast cancer since they can be utilized as biomarkers to determine initiation, progression, and metastasis, or as potential therapeutic targets ${ }^{7}$ since they apply resistance toward chemotherapeutic agents, through the activation of the same signaling pathways in the different subtypes of breast cancer, using different targets. Next, there are some examples of IncRNAs (Table 2).

\section{Urothelial cancer associated 1 (UCA1)}

Its expression is regulated by the hypoxia-inducible factor 1-alpha (HIF-1A) transcription factor. This IncRNA presents a high expression in breast cancer, after its exposure to tamoxifen giving rise to the activation of the $A K T / m T O R$ and $W n t / \beta$-catenin signaling pathways. Wnt/ $\beta$-catenin pathway activation prevents the degradation of $\beta$-catenin, translocating it to the nucleus, allowing the redistribution of the RE and the preservation of the characteristics of stem cells. Moreover, this IncRNA inhibits the expression of p27 (cell cycle regulator) and miR-18 ${ }^{2}$ (microRNA 18s); the latter acts as a tumor suppressor when joined to the 3'-UTR (untranslated region) of HIF-1A (preventing it from joining its targets). Under normal conditions, miR-18a inhibits the Yes-associated protein 1 (YAP1), which positively regulates cell division cycle 42 , which is a modulator of the cell cycle, and in breast cancer is involved in increasing proliferation, survival, and metastasis through the phosphatidylinositol 3-kinase (PI3K) pathway and mitogen-activated protein kinases 
Table 2. Summary of the roles of IncRNAs involved in breast cancer

\begin{tabular}{|c|c|c|c|c|}
\hline IncRNAs & $\begin{array}{l}\text { Expression in } \\
\text { breast cancer }\end{array}$ & Role in breast cancer & Pathways & Drug resistance \\
\hline UCA1 & Elevated & $\begin{array}{l}\text { Maintenance of carcinogenic stem cells, increases } \\
\text { proliferation, survival, and metastasis in cancer cells }\end{array}$ & $\begin{array}{l}\text { AKT } / \mathrm{mTOR} \\
\text { Wnt } / \beta \text {-catenin } \\
\text { MAPK and HIFA }\end{array}$ & $\begin{array}{l}\text { Tamoxifen } \\
\text { Trastuzumab }\end{array}$ \\
\hline LINK-A & Elevated & $\begin{array}{l}\text { Modifications in metabolism, greater energy disposition, } \\
\text { and potency of tumorigenesis }\end{array}$ & $\begin{array}{l}\text { Glycolysis and } \\
\text { HIF1A }\end{array}$ & $\begin{array}{l}\text { Endocrine drugs and } \\
\text { trastuzumab }\end{array}$ \\
\hline NEAT1 & Elevated & $\begin{array}{l}\text { Increases tumor size, metastasis, low survival in patients, } \\
\text { EMT, avoids translation and hijacks transcripts that are } \\
\text { responsible for regulating the cell cycle }\end{array}$ & $\begin{array}{l}\text { HIF2A and } \\
\text { WNT }\end{array}$ & $\begin{array}{l}\text { Endocrine treatments } \\
\text { and 5-flurocyl }\end{array}$ \\
\hline HOTAIR & Elevated & $\begin{array}{l}\text { Low survival in patients; promotes migration } \\
\text { angiogenesis, invasion, and metastasis in cells. }\end{array}$ & $\begin{array}{l}\text { PI3K/AKT and } \\
\text { HIF1A }\end{array}$ & Tamoxifen \\
\hline BCAR4 & Elevated & Helps cell proliferation and tumorigenesis & AKT/ERK & Tamoxifen \\
\hline GAS5 & Low & Formation of tumors and altering the cellular metabolism & AKT/PI3K & Tamoxifen \\
\hline MA-Linc1 & Elevated & $\begin{array}{l}\text { Interrupts mitosis and destabilizes microtubules, } \\
\text { promoting survival }\end{array}$ & $\begin{array}{l}\text { Apoptosis and } \\
\text { cell cycle }\end{array}$ & Taxol \\
\hline MAPT-AS & Elevated & $\begin{array}{l}\text { Stabilizes the transcript encoding TAU and prevents cell } \\
\text { death }\end{array}$ & ------- & Taxol \\
\hline
\end{tabular}

IncRNAs: long non-coding RNAs; UCA1: urothelial cancer-associated 1; NEAT1: nuclear paraspeckle assembly transcript 1; H0TAIR: hox transcript antisense RNA; GAS5: growth arrest-specific 5; MAPT-AS: microtubule-associated protein tau antisense RNA; EMT: epithelial-mesenchymal transition.

pathways $^{29}$. Therefore, UCA1 plays an oncogenic role in breast cancer, allowing the creation of resistance against tamoxifen and trastuzumab (elevated expression of YAP1) $)^{30,31}$.

\section{Long intergenic non-protein coding RNA 1139 (LINK-A)}

It is a cytoplasmic IncRNA with elevated expression in triple-negative breast cancer. It induces the HIF-1A signaling pathway under normoxic conditions, through the heterodimerization of epidermal-type growth factor bound to heparin dependent to the glycoprotein non-metastatic protein b (EGDR), giving rise to LINK-A recruitment, which interacts with the breast tumor kinase (protein tyrosine kinase) and leucine-rich repeat kinase, causing the phosphorylation of HIF-1A in its tyrosine 565 and serine 797, respectively. Phosphorylated serine 797 interacts with p300 (the regulator of the remodeling of chromatin), setting off the activation of the glycolysis pathway under normoxic conditions ${ }^{7,32}$ and enhancing tumorigenesis.

\section{NEAT1}

NEAT1 expression is regulated by the HIF-2A transcription factor, instead of HIF-1A (UCA1) ${ }^{33}$; its high expression is correlated with the size of the tumor and its metastatic potential, linked to a low survival rate. NEAT1 interacts with the forkhead box N3 repressor complexes and SIN3A (SIN3 transcriptional regulator family member A), which inhibit GATA3 (GATA binding protein 3 is a transcription factor which inhibits epithelial-mesenchymal transition [EMT]), allowing $\mathrm{EMT}^{7}$ in positive cells for the ER. Moreover, an elevated expression of NEAT1 increases the activity of the WNT signaling pathways, regulating ERs, and resistance to endocrine treatments such as the case of the UCA1 IncRNA. On the other hand, NEAT1 blocks the miR-211 function (tumor suppressor), allowing the expression of (high mobility group AT-hook [HMGA] which is non-histone proteins with transcriptional activity) and promoting EMT (miR-211 inhibits HMGA expression by binding itself in its region $3^{\prime}$ ), causing resistance to the 5-fluorouracil agent (analog of uracil, with an atom of flour in carbon 5$)^{34}$, which inhibits the normal functions of DNA and $\mathrm{RNA}^{35}$. In addition, paraspeckles also play an important role in the proliferation and reduction of apoptosis in breast cancer, through kidnapping and editing (avoiding its translation) of (F11 receptor [F11R], which is an adhesion molecule) under conditions of hypoxia ${ }^{33}$. 


\section{Hox transcript antisense RNA (HOTAIR)}

It is a low survival biomarker and the regulation of its expression depends on the transcription factors HIF1A, AP1 (jun proto-oncogenes), SP1 (Sp1 transcription factor), nuclear factor kappa $B$, and binding sites for estrogen response elements (EREs). The RE in the absence of estrogen interacts with ERE (ligand RE) and induces changes in chromatin through histone methyltransferase (MLL1 and MLL3), for the transcriptional activation of the HOTAIR gene, in cells positive to $\mathrm{RE}^{7,30}$. Moreover, HOTAIR produces positive feedback when inducing the RE expression ${ }^{36}$. Furthermore, in the presence of estrogen, HOTAIR expression decreases and growth regulating ER binding 1 expression increases. This is because the RE-estrogen complex induces a loop between the site of transcription start and enhancer of the HOTAIR gene, preventing its expression ${ }^{30,36}$. HOTAIR alters the expression of its targets through its 5' and 3' regions, which join to PRC2 (transcriptional repression) and LSD1 (lysine demethylase $1 \mathrm{~A}$, which activates transcription), respectively ${ }^{31}$. Through its 5' and 3' regions, HOTAIR regulates the interaction of epithelial cells, increasing ATAXIN-1 degradation, which modulates E-cadherin transcription, promoting EMT, and also promoting metastasis and an increase of the HER 2 expression when bound to miR 331-3p (tumor suppressor) ${ }^{7}$. As for the induction of overexpression of HOTAIR by HIF1A in conditions of hypoxia, an elevated expression of $A X L$ (tyrosine kinase receptor) is observed, promoting survival, migration, angiogenesis, invasion, and metastasis through signaling cascade of PI3K/AKT ${ }^{37}$ in African women with breast cancer ${ }^{38}$.

\section{Growth arrest-specific 5}

Reduces the efficacy of tamoxifen therapy through an increasing in phosphorylation of the ERBB2 and ERBB3 oncogenes, which are regulators of the AKT and ERK (1/2) signaling pathway ${ }^{39}$. Furthermore, an amplification and overexpression of the HER2 receptor on the surface of the cells is exhibited, inducing the activation of the RE AIB-1 / SRC-3 coactivator and causing cell proliferation and tumorigenesis ${ }^{40}$. In normal conditions (not cancer), GAS5 is a regulator of the metabolism, survival, and apoptotic stimuli; since it competes with the glucocorticoid receptor to bind to the glucocorticoid response elements ${ }^{41}$. Unlike other IncRNAs, this is subexpressed, correlating with a bad prognosis and creating resistance against trastuzumab.
Its low expression causes its target, miR-21, presents a high expression, inducing the formation of tumors through an accelerated proliferation and cellular invasion. Furthermore, this micro-RNA inhibits the expression of phosphatase and tensin homolog, which negatively regulates the AKT/PI3K pathway ${ }^{42}$.

\section{Mitosis-associated long intergenic non-coding RNA 1 (MA-linc1) and microtubule-associated protein tau (MAPT) antisense RNA 1 (MAPT-AS1)}

These IncRNAs evade the response to taxol, which is a stabilizing agent of the microtubules, preventing entry into mitosis and causing cellular death ${ }^{43}$. MA-linc1 regulates the cell cycle, leading to the finalization of mitosis, through the destabilization of microtubules ${ }^{31}$. On the other hand, MAPT-AS1 is an antisense transcript of the MAPT gene, which encodes for TAU protein, which, in turn, competes with taxol to bind to microtubules. MAPT-AS1 binds to the MAPT transcript and stabilizes it to produce high levels of TAU ${ }^{44}$.

\section{Resistance acquisition mechanisms}

Patients with cancer initially respond quite well to prescribe chemotherapeutic agents, but once they create resistance, survival prognosis lowers ${ }^{45-47}$. A key factor for resistance is the large intratumor heterogeneity since it is a combination of stem cells, progenitor cells, and its progeny, providing the tumor with very variable cell phenotypes, and can even be present in different subtypes of breast cancer. This idea is supported by the hypothesis that luminal subtypes and HER2 originate from progenitor cells committed to the luminal lineage, while the basal subtype comes from less differentiated stem cells, and in some cases, progenitor cells committed to luminal lineage can be precursors for the development of the basal subtype, through epigenetic and genetic changes ${ }^{28,48-50}$ (Fig. 4).

Other causes that add intratumor heterogeneity can be epigenetic changes and mutations in the genome, through parallel evolution, which allows the cells to present very diverse expression patterns that through positive selective pressure are selected and present in coding or non-coding regions (regulatory elements) ${ }^{51}$. These changes are transmitted to the progeny during cellular splitting ${ }^{52}$ (Fig. 5) promoting resistance toward chemotherapeutic agents.

Mutations of the genome lead to permanent changes of the DNA, while epigenetic alterations are transitory 


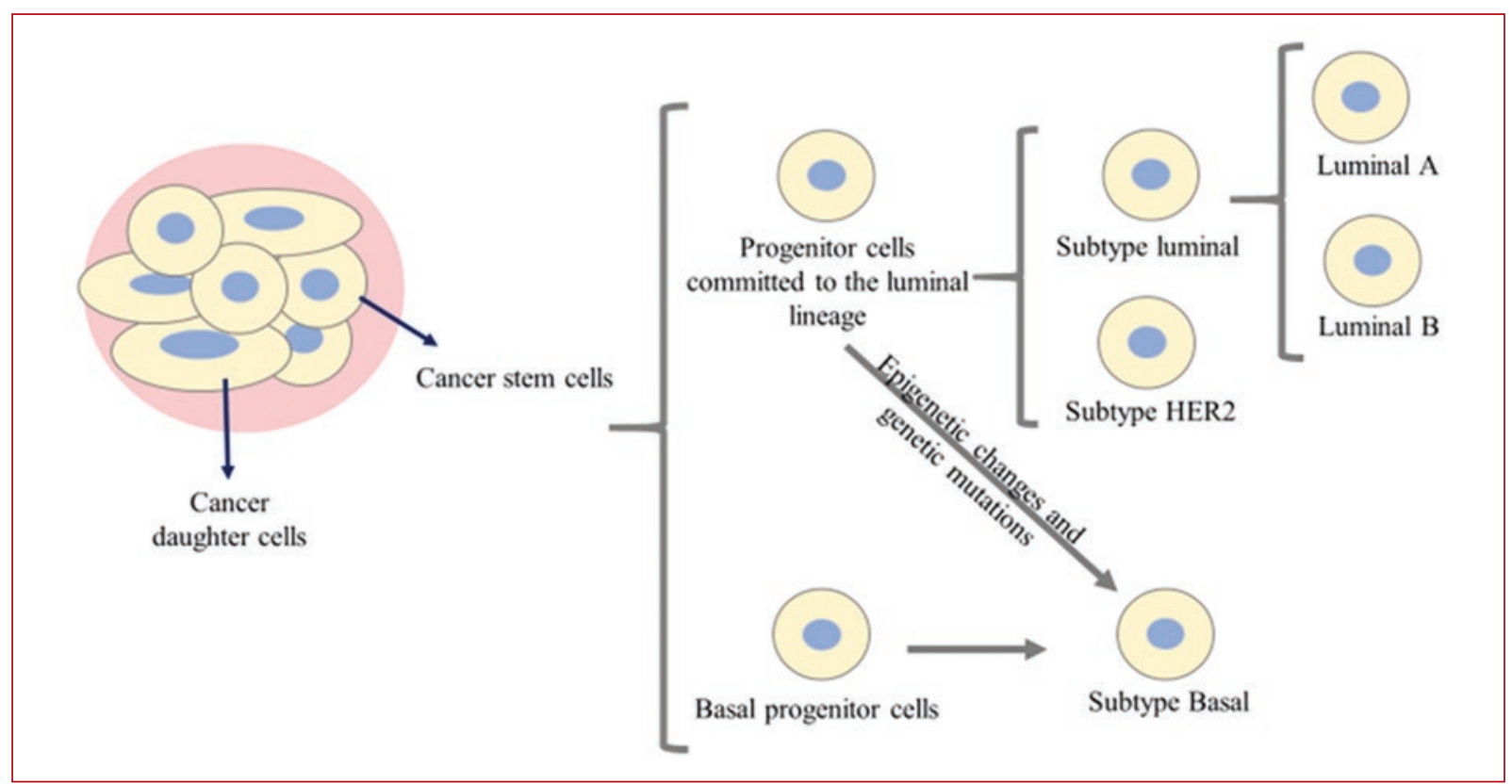

Figure 4. Hypothesis of the development of the different subtypes of breast cancer. The cancer stem cells are capable of giving rise to slightly more differentiated stem cells such as progenitor cells committed to the luminal lineage and basal, allowing the development of the luminal-human epidermal growth factor receptor 2 and basal subtypes, respectively. However, sometimes, the progenitor cells committed to the luminal lineage are able to give rise to the basal subtype through epigenetic and genetic changes.

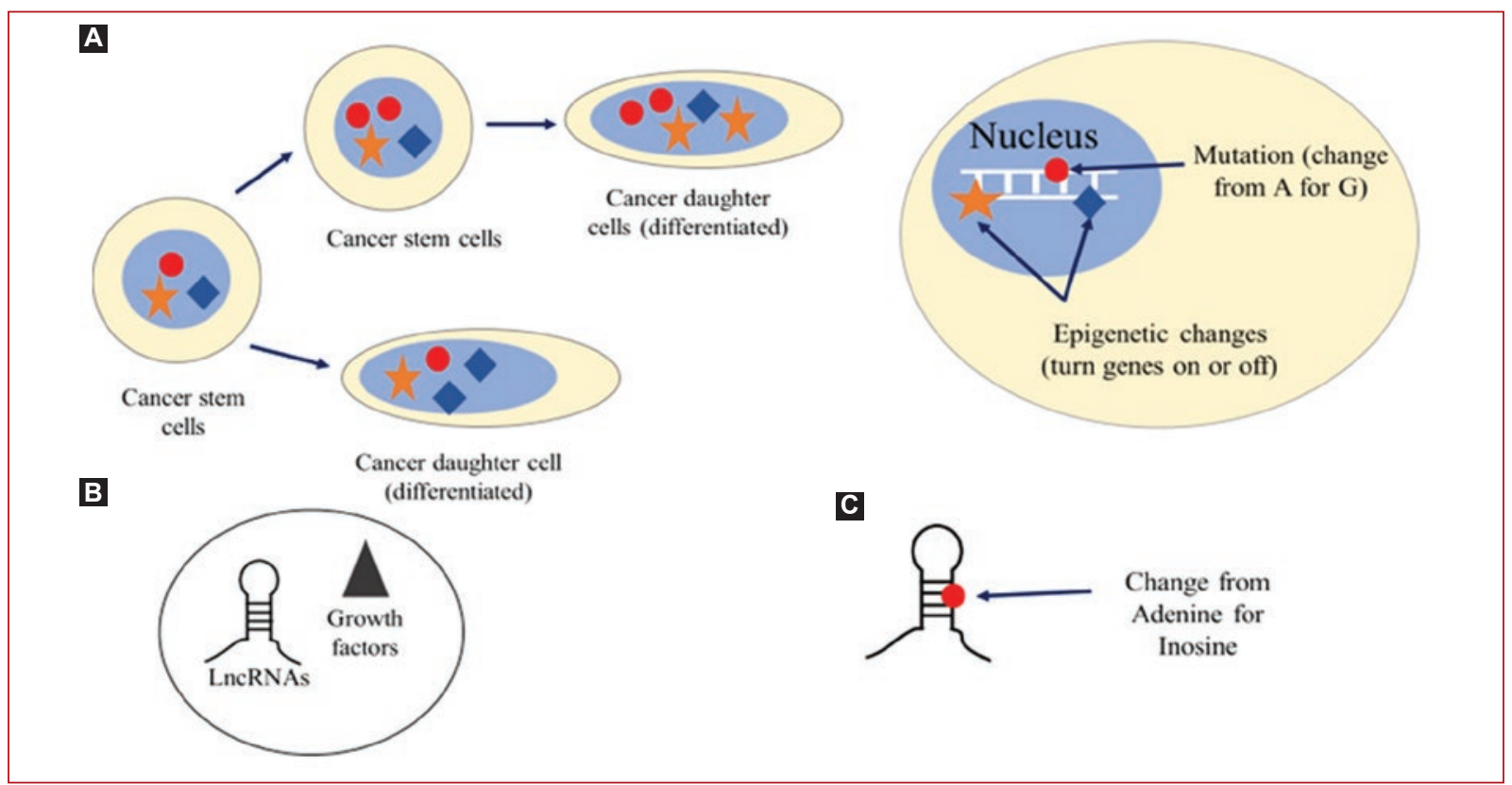

Figure 5. Mechanisms of resistance. Stem and daughter cells can use different mechanisms to develop resistance to various chemotherapeutic agents, among them $\mathbf{A}$ : parallel evolution, in which the stem cell passes on its mutations and epigenetic changes during cellular division to its daughter cells, whether stem cells or differentiated cells, which are capable of acquiring new mutations and epigenetic changes and transmitting them to their offspring. B: Another mechanism is the exosomes, which are compartments that carry IncRNAs and proteins in other molecules, and is able to be transported to other cells, allowing the development of resistance. C: Finally, there is hyperedition, of which we do not know much yet, but it is proposed that it can confer transcripts with certain characteristics that allow it to evade the action of chemotherapeutic drugs. 
changes; the latter allows the cell to have a greater cellular plasticity without compromising the genotype. Studies have proven that resistance mechanisms can have a base in genetic or epigenetic changes.

Xue et al. ${ }^{53}$ demonstrated genetic-based changes. Through a model of mice with xenografts derived from patients who were carriers of the B-Raf mutation (BRAFV600E allows the expression of the ERK pathway without external stimuli) $)^{54}$. The model was administered different inhibitors of the ERK signaling pathway, and the emergence of extra chromosome material was observed through sequencing of a single cell and fluorescent hybridization in situ, which is believed to have arisen through parallel evolution shortly after treatment; the selective pressure of cells continuously treated with a single medication allowed the tumor cells to adapt while maintaining their intratumor heterogeneity ${ }^{53}$. Regarding IncRNAs, HOTAIR is a clear example which is amplified in the genome, promoting resistance to tamoxifen ${ }^{55}$.

Sharma et al. ${ }^{56}$ proved a resistance with a base in epigenetic changes and also that this can be reverted. To do so, PC9 cells were incubated (cells derived from lung cancer, sensitive to inhibitors of EGFR-receptor epidermal growth factor) with inhibitors of the EGFR, inducing death in most of the cellular population, with only a small fraction surviving, which presented resistance toward the inhibitors. To determine whether these changes were genetic or epigenetic, the surviving cells were incubated with deacetylase histone inhibitors, causing cellular death after losing their resistance to the inhibitors of EGFR. On the other hand, Vaz et al. ${ }^{57}$ proved that the environment induces epigenetic changes through exposure to bronchial epithelial cells (non-cancerous) to condensed cigarette smoke, causing epigenetic changes and inducing the transformation of wild-mutated KRAS (KRAS proto-oncogene, GTPase), revealing that progressive exposure to condensed cigarette smoke induces changes in the methylation of the DNA, and promoting pro-oncogenic changes. Concerning the IncRNAs, HOTAIR is a clear example of epigenetic changes caused by external stimuli or chemical agents like estradiol. The expression of HOTAIR is found to be elevated by its interaction with estradiol through its ERE-binding sites (in the promoter), modifying the structure of chromatin, and activating genes related to cellular proliferation, inhibition of apoptosis and EMT ${ }^{58}$.

Another resistance mechanism is exosomes, which are vesicles that carry diverse RNAs and proteins. Xu et al. ${ }^{59}$ proved that the cellular line MCF-7 (sensitive to tamoxifen), showed resistance to tamoxifen when treated with exosomes originated from the cellular line LCC2 (resistant to tamoxifen). Said exosomes presented a high expression of UCA1, causing proliferation, survival, metastasis, and resistance to tamoxifen and trastuzumab (Fig. 5) $)^{30,31,59}$.

Moreover, IncRNAs can be hyperedited, which is suggested is also a key component of the transcriptional diversity in tumors ${ }^{60}$. Hyperedition can lead to the loss of functions in IncRNAs ${ }^{61}$, mainly in those who have the function of tumor suppressors $(\mathrm{F} 11 \mathrm{R})^{33}$. However, there is still a need for studies to determine if it plays an important role in resistance toward chemotherapeutic medications (Fig. 5).

Therefore, genetic changes like amplifications in the genome, together with the epigenetic alterations (which occur in genes which transcribe IncRNAs or in genes which are regulated by them), exosomes, and hyperedition give breast cancer cells distinctive characteristics (hallmarks). These provide them with advantages compared to normal tissue, such as the activation of signaling pathways related to cellular proliferation; evasion of growth suppressors, resistance to cellular death, cell replication capacity, induction to angiogenesis, activation of invasion (cellular metastasis), cellular metabolism reprogramming, and evasion to immune response ${ }^{62}$. Thus, cancerous cells present the same phenotype since the same signaling pathways are activated through synonym inductors, causing resistance to different therapeutic agents.

\section{Strategies used to determine IncRNA functions}

Studying the function of the IncRNAs implicated in breast cancer is critical, yet it remains a challenge because the bioinformatic tools do not allow us to determine the secondary structure and post-transcriptional modifications which it may suffer (hyperedition), altering the binding to its targets. Hence, the most viable methods nowadays are studies of loss/gain of function in in vivo or in vitro models.

\section{Loss of function}

RNA interference is utilized in this type of analysis to block the expression, but the inhibition is temporal. Therefore, the loss of function is transitory. Clustered regularly interspaced short palindromic repeats, associated protein 9 (CRISPR-Cas9), is a promising tool that allows the explanation of IncRNA functions, performing editions in the genome, either generating a partial or total deletion of the IncRNAs, or blocking the 
expression from the alteration of the sequencings located between the promoter and the sequence of interest, through the insertion of polyadenylation signals ${ }^{63}$.

\section{Gain-of-function studies}

The use of CRISPR-Cas9 also allows the overexpression of our sequence of interest due to the insertion of a strong promoter upstream the gene ${ }^{64}$. However, the most used techniques for these studies are the use of plasmids or adenovirus carriers of the sequence of interest, being the overexpression transitory ${ }^{65,66}$.

\section{In vivo and in vitro models}

Concerning the in vivo models, it is still difficult to establish an animal model, due to the high evolution rate of the primary sequence, among the species (activating targets and different pathways between species). Moreover, certain IncRNAs present in humans do not exist in other mammals. On the other hand, the use of cellular lines as an in vitro model does not provide us with accurate information, which can extrapolate patients since cellular lines present a high instability in its genomic and epigenetic architecture, which differs in large proportion to the tumor from which it derived. In addition, 3D cultures are needed and the use of diverse cellular populations that interact in normal conditions. Furthermore, the technologies used (expression microarrangements and sequencing) to analyze the function or targets of IncRNAs are very varied, causing inconsistencies among studies when analyzing the data ${ }^{7}$. Therefore, bioinformatics programs are needed, which will allow us to predict the function and possible modifications that IncRNAs may suffer.

\section{Conclusion}

LncRNAs can be used as therapeutic targets or biomarkers since they play an important role in the proliferation, metastasis, migration, and evasion of the actions of drugs in cancerous cells, promoting the aggressiveness of the tumor and causing a low survival rate. Moreover, IncRNAs intervene in the development of resistance mechanisms through the activation of the same signaling pathways in the different subtypes of breast cancer, either participating as an intermediary or stimulating the activation of transcription factors essential for the expression of the genes necessary for the activation of signaling pathways. Further studies of IncRNAs are needed, regarding the modifications performed by the hyperedition and determining whether this process is linked to the development of resistance toward different chemotherapeutic agents and the presence of more aggressive breast tumors.

\section{Conflicts of interest}

The authors do not have conflicts of interest to declare.

\section{Funding}

EA Flores-Contreras was supported by a CONACYT scholarship.

\section{Acknowledgments}

The authors would like to thank CONACYT for scholarship support and the Cytogenomics and Microarray Laboratory for providing space and computer equipment for the development of this review article.

\section{Ethical disclosures}

Protection of human and animal subjects. The authors declare that no experiments were performed on humans or animals for this study.

Confidentiality of data. The authors declare that they have followed the protocols of their work center on the publication of patient data.

Right to privacy and informed consent. The authors declare that no patient data appear in this article.

\section{References}

1. Rijnkels M, Kabotyanski E, Montazer-Torbati MB, Hue Beauvais C, Vassetzky $Y$, Rosen JM, et al. The epigenetic landscape of mammary gland development and functional differentiation. J Mammary Gland Biol Neoplasia. 2010;15:85-100.

2. Campos El, Reinberg D. Histones: annotating chromatin. Annu Rev Genet. 2009;43:559-99.

3. Dhasarathy A, Wade PA. The MBD protein family-reading an epigenetic mark? Mutat Res. 2008;647:39-43.

4. Postnikov $Y$, Bustin M. Regulation of chromatin structure and function by HMGN proteins. Biochim Biophys Acta. 2010;1799:62-8.

5. Mattick JS, Amaral PP, Dinger ME, Mercer TR, Mehler MF. RNA regulation of epigenetic processes. Bioessays. 2009;31:51-9.

6. Kouzarides T. Chromatin modifications and their function. Cell. 2007;128:693-705.

7. Richard JL, Eichhorn PJ. Deciphering the roles of IncRNAs in breast development and disease. Oncotarget. 2018;9:20179-212.

8. Macias H, Hinck L. Mammary gland development. Wiley Interdiscip Rev Dev Biol. 2012;1:533-57.

9. Hassiotou F, Geddes D. Anatomy of the human mammary gland: current status of knowledge. Clin Anat. 2013;26:29-48

10. Hangauer MJ, Vaughn IW, McManus MT. Pervasive transcription of the human genome produces thousands of previously unidentified long intergenic noncoding RNAs. PLoS Genet. 2013;9:e1003569.

11. Iyer MK, Niknafs YS, Malik R, Singhal U, Sahu A, Hosono Y, et al. The landscape of long noncoding RNAs in the human transcriptome. Nat Genet. 2015;47:199-208.

12. Derrien T, Johnson R, Bussotti G, Tanzer A, Djebali S, Tilgner H, et al. The GENCODE $v 7$ catalog of human long noncoding RNAs: analysis of 
their gene structure, evolution, and expression. Genome Res. 2012 22:1775-89

13. Guttman M, Amit I, Garber M, French C, Lin MF, Feldser D, et al. Chromatin signature reveals over a thousand highly conserved large non-coding RNAs in mammals. Nature. 2009;458:223-7.

14. Kapranov P, Cheng J, Dike S, Nix DA, Duttagupta R, Willingham AT, et al. RNA maps reveal new RNA classes and a possible function for pervasive transcription. Science. 2007;316:1484-8.

15. Huarte $M$. The emerging role of IncRNAs in cancer. Nat Med. 2015;21:1253-61.

16. Lanzós A, Carlevaro-Fita J, Mularoni L, Reverter F, Palumbo E, Guigó R et al. Discovery of cancer driver long noncoding RNAs across 1112 tumour genomes: new candidates and distinguishing features. Sc Rep. 2017;7:41544

17. Ponjavic J, Ponting CP, Lunter G. Functionality or transcriptional noise? Evidence for selection within long noncoding RNAs. Genome Res. 2007; $17: 556-65$

18. Nitsche A, Stadler PF. Evolutionary clues in IncRNAs. Rev Wiley Interdiscip Rev. 2016;8:1376-89.

19. Gooding AJ, Zhang B, Jahanbani FK, Gilmore HL, Chang JC Valadkhan S, et al. The IncRNA BORG drives breast cancer metastasis and disease recurrence. Sci Rep. 2017;7:12698.

20. Ponting CP, Oliver PL, Reik W. Evolution and functions of long noncoding RNAs. Cell. 2009;136:629-41.

21. Standaert L, Adriaens C, Radaelli E, Van Keymeulen A, Blanpain C, Hirose $T$, et al. The long noncoding RNA neat 1 is required for mammary gland development and lactation. RNA. 2014;20:1844-9.

22. Askarian-Amiri ME, Crawford J, French JD, Smart CE, Smith MA Clark MB, et al. SNORD-host RNA zfas1 is a regulator of mammary development and a potential marker for breast cancer. RNA. 2011;17:878-91.

23. Ginger MR, Gonzalez-Rimbau MF, Gay JP, Rosen JM. Persistent changes in gene expression induced by estrogen and progesterone in the rat mammary gland. Mol Endocrinol. 2001;15:1993-2009.

24. Hansji H, Leung EY, Baguley BC, Finlay GJ, Cameron-Smith D, Figueiredo VC, et al. ZFAS1: a long noncoding RNA associated with ribosomes in breast cancer cells. Biol Direct. 2016;11:62.

25. Shore AN, Kabotyanski EB, Roarty K, Smith MA, Zhang Y, Creighton CJ, et al. Pregnancy-induced noncoding RNA (PINC) associates with polycomb repressive complex 2 and regulates mammary epithelial differentiation. PLOS Genet. 2012;8:e1002840.

26. Su X, Malouf GG, Chen Y, Zhang J, Yao H, Valero V, et al. Comprehensive analysis of long non-coding RNAs in human breast cancer clinical subtypes. Oncotarget. 2014;5:9864-76.

27. Slamon DJ, Leyland-Jones B, Shak S, Fuchs H, Paton V, Bajamonde A, et al. Use of chemotherapy plus a monoclonal antibody against HER2 for metastatic breast cancer that overexpresses HER2. N Engl J Med. 2001;344:783-92.

28. Polyak K. Heterogeneity in breast cancer. J Clin Invest. 2011;121:3786-8.

29. Sakabe M, Fan J, Odaka Y, Liu N, Hassan A, Duan X, et al. YAP/TAZ CDC42 signaling regulates vascular tip cell migration. Proc Natl Acad Sci U S A. 2017;114:10918-23.

30. Malhotra A, Jain M, Prakash $H$, Vasquez KM, Jain A. The regulatory roles of long non-coding RNAs in the development of chemoresistance in breast cancer. Oncotarget. 2017:8:110671-84.

31. Campos-Parra AD, López-Urrutia E, Orozco Moreno LT, López-Camarillo C, Meza-Menchaca T, Figueroa González G, et al. Long non-coding RNAs as new master regulators of resistance to systemic treatments in breast cancer. Int J Mol Sci. 2018;19:E2711.

32. Choudhry $\mathrm{H}$, Harris AL. Advances in hypoxia-inducible factor biology. Cell Metab. 2018:27:281-98

33. Choudhry H, Albukhari A, Morotti M, Haider S, Moralli D, Smythies J, et al. Tumor hypoxia induces nuclear paraspeckle formation through HIF-2 $\alpha$ dependent transcriptional activation of NEAT1 leading to cancer cell survival. Oncogene. 2015;34:4482-90.

34. Li X, Wang S, Li Z, Long X, Guo Z, Zhang G, et al. The IncRNA NEAT1 facilitates cell growth and invasion via the miR-211/HMGA2 axis in breast cancer. Int J Biol Macromol. 2017; 105:346-53.

35. Longley DB, Harkin DP, Johnston PG 5-fluorouracil: mechanisms of action and clinical strategies. Nat Rev Cancer. 2003;3:330-8.

36. Xue X, Yang YA, Zhang A, Fong KW, Kim J, Song B, et al. LncRNA HOTAIR enhances ER signaling and confers tamoxifen resistance in breast cancer. Oncogene. 2016;35:2746-55

37. Hong Q, Li O, Zheng W, Xiao WZ, Zhang L, Wu D, et al. LncRNA HOTAIR regulates HIF-1 $\alpha / A X L$ signaling through inhibition of miR-217 in renal cell carcinoma. Cell Death Dis. 2017:8:e2772.

38. Nalwoga $H$, Ahmed L, Arnes JB, Wabinga H, Akslen LA. Strong expression of hypoxia-inducible factor- $1 \alpha$ (HIF-1 $\alpha$ ) is associated with axl expression and features of aggressive tumors in african breast cancer. PLoS One. 2016;11:e0146823.

39. Godinho MF, Sieuwerts AM, Look MP, Meijer D, Foekens JA, Dorssers LC et al. Relevance of BCAR4 in tamoxifen resistance and tumour aggressiveness of human breast cancer. $\mathrm{Br} \mathrm{J}$ Cancer. 2010;103:1284-91.
40. Osborne CK, Bardou V, Hopp TA, Chamness GC, Hilsenbeck SG, Fuqua SA, et al. Role of the estrogen receptor coactivator AIB1 (SRC-3) and HER-2/neu in tamoxifen resistance in breast cancer. J Natl Cancer Inst. 2003;95:353-61.

41. Kino $\mathrm{T}$, Hurt DE, Ichijo $\mathrm{T}$, Nader $\mathrm{N}$, Chrousos GP. Noncoding RNA gas 5 is a growth arrest and starvation-associated repressor of the glucocorticoid receptor. Sci Signal. 2010;3:ra8.

42. Li W, Zhai L, Wang H, Liu C, Zhang J, Chen W, et al. Downregulation of IncRNA GAS5 causes trastuzumab resistance in breast cancer. Oncotarget. 2016;7:27778-86.

43. Wang F, Porter M, Konstantopoulos A, Zhang P, Cui H. Preclinical development of drug delivery systems for paclitaxel-based cancer chemotherapy. J Control Release. 2017;267:100-18.

44. Pan Y, Pan Y, Cheng Y, Yang F, Yao Z, Wang O, et al. Knockdown of LncRNA MAPT-AS1 inhibites proliferation and migration and sensitizes cancer cells to paclitaxel by regulating MAPT expression in ER-negative breast cancers. Rev Cell Biosci. 2018;8:7.

45. Santa-Maria CA, Gradishar WJ. Changing treatment paradigms in metastatic breast cancer: Lessons learned. JAMA Oncol. 2015;1:528-34.

46. Salgia R. Mutation testing for directing upfront targeted therapy and post-progression combination therapy strategies in lung adenocarcinoma. Expert Rev Mol Diagn. 2016;16:737-49.

47. Lopez JS, Banerji U. Combine and conquer: challenges for targeted therapy combinations in early phase trials. Nat Rev Clin Oncol. 2017; 14:57-66.

48. Chaffer CL, Brueckmann I, Scheel C, Kaestli AJ, Wiggins PA, Rodrigues LO, et al. Normal and neoplastic nonstem cells can spontaneously convert to a stem-like state. Proc Natl Acad Sci U S A. 2011; 108:7950-5.

49. Proia TA, Keller PJ, Gupta PB, Klebba I, Jones AD, Sedic M, et al Genetic predisposition directs breast cancer phenotype by dictating progenitor cell fate. Cell Stem Cell. 2011;8:149-63.

50. Lim E, Vaillant F, Wu D, Forrest NC, Pal B, Hart AH, et al. Aberrant luminal progenitors as the candidate target population for basal tumor development in BRCA1 mutation carriers. Nat Med. 2009;15:907-13.

51. Jaenisch R, Bird A. Epigenetic regulation of gene expression: how the genome integrates intrinsic and environmental signals. Nat Genet. 2003;33 Suppl:245-54

52. Berger SL, Kouzarides T, Shiekhattar R, Shilatifard A. An operational definition of epigenetics. Genes Dev. 2009;23:781-3.

53. Xue Y, Martelotto L, Baslan T, Vides A, Solomon M, Mai TT, et al. An approach to suppress the evolution of resistance in BRAFV600E mutant cancer. Nat Med. 2017;23:929-37.

54. Cantwell-Dorris ER, O'Leary JJ, Sheils OM. BRAFV600E: implications for carcinogenesis and molecular therapy. Mol Cancer Ther. 2011;10:385-94.

55. Xu S, Kong D, Chen Q, Ping Y, Pang D. Oncogenic long noncoding RNA landscape in breast cancer. Mol Cancer. 2017;16:129.

56. Sharma SV, Lee DY, Li B, Quinlan MP, Takahashi F, Maheswaran S, et al. A chromatin-mediated reversible drug-tolerant state in cancer cell subpopulations. Cell. 2010;141:69-80.

57. Vaz M, Hwang SY, Kagiampakis I, Phallen J, Patil A, O'Hagan HM, et al. Chronic cigarette smoke-induced epigenomic changes precede sensitization of bronchial epithelial cells to single-step transformation by KRAS mutations. Cancer Cell. 2017;32:360-76000000.

58. Bhan A, Hussain I, Ansari KI, Bobzean SA, Perrotti LI, Mandal SS, et al. Bisphenol-A and diethylstilbestrol exposure induces the expression of breast cancer associated long noncoding RNA HOTAIR in vitro and in vivo. J Steroid Biochem Mol Biol. 2014;141:160-70.

59. Xu CG, Yang MF, Ren YQ, Wu CH, Wang LQ. Exosomes mediated transfer of IncRNA UCA1 results in increased tamoxifen resistance in breast cancer cells. Eur Rev Med Pharmacol Sci. 2016;20:4362-8.

60. Paz-Yaacov N, Bazak L, Buchumenski I, Porath HT, Danan-Gotthold M, Knisbacher BA, et al. Elevated RNA editing activity is a major contributor to transcriptomic diversity in tumors. Cell Rep. 2015;13:267-76.

61. Yang $Y$, Zhou X, Jin Y. ADAR-mediated RNA editing in non-coding RNA sequences. Sci China Life Sci. 2013:56:944-52.

62. Hanahan D, Weinberg RA. Hallmarks of cancer: the next generation. Cell. 2011;144:646-74.

63. Eißmann M, Gutschner T, Hämmerle M, Günther $S$, Caudron-Herger $M$, Groß M, et al. Loss of the abundant nuclear non-coding RNA MALAT1 is compatible with life and development. RNA Biol. 2012;9:1076-87.

64. Xiang JF, Yin QF, Chen T, Zhang Y, Zhang XO, Wu Z, et al. Human colorectal cancer-specific CCAT1-L IncRNA regulates long-range chromatin interactions at the MYC locus. Cell Res. 2014;24:513-31.

65. Ho TT, Zhou N, Huang J, Koirala P, Xu M, Fung R, et al. Targeting non-coding RNAs with the CRISPR/Cas9 system in human cell lines. Nucleic Acids Res. 2015;43:e17.

66. Han J, Zhang J, Chen L, Shen B, Zhou J, Hu B, et al. Efficient in vivo deletion of a large imprinted IncRNA by CRISPR/Cas9. RNA Biol. 2014;11:829-35 